\title{
Insight into surgical outcomes of post infarct-ventricular septal defect repair through a 23-years retrospective study-invited commentary
}

Doniparthi Pradeep ${ }^{1}$

${ }^{1}$ All India Institute of Medical Sciences

November 2, 2021

\begin{abstract}
The authors present an excellent retrograde analysis of a rare condition of a phenomenal number of cases and their surgical outcomes. A majority of the studies in published literature are anecdotal case reports which are a rare and dreadful entity. A comprehensive countrywide view of the UK National Adult Cardiac Surgery Audit database is presented in this study. This study represents the changing trends in the risk factors, management strategies, and outcomes of ventricular septal rupture for over 23 years in a nutshell.
\end{abstract}

Insight into surgical outcomes of post infarct-ventricular septal defect repair through a 23-years retrospective study-invited commentary

Running Title: Ventricular septal rupture in a nutshell

Doniparthi Pradeep, MS ${ }^{1}$

Department of Cardiothoracic and Vascular Surgery

All India Institute of Medical Sciences, New Delhi ${ }^{1}$

Correspondence:

Dr.Doniparthi Pradeep, MS

Senior Resident

Department of Cardiothoracic and Vascular Surgery

AIIMS, New Delhi-110029, INDIA

Ph: +91-9501626593

Email:pradeepdhoni023.pd@gmail.com

Orcid ID: https://orcid.org/0000-0002-3693-7271

Insight into surgical outcomes of post infarct-ventricular septal defect repair through a 23-years retrospective study-invited commentary

Abstract:

The authors present an excellent retrograde analysis of a rare condition of a phenomenal number of cases and their surgical outcomes. A majority of the studies in published literature are anecdotal case reports 
which are a rare and dreadful entity. A comprehensive countrywide view of the UK National Adult Cardiac Surgery Audit database is presented in this study. This study represents the changing trends in the risk factors, management strategies, and outcomes of ventricular septal rupture for over 23 years in a nutshell.

\section{Commentary:}

This paper holds a rather exclusive position in the domain of cardiac surgery; representing a rare, lethal, and mortal condition in a large number, 1010 cases, and spanning over 23 years, representing a single population. Ventricular septal rupture (VSR) is known to cause extensive damage to the myocardium with shunting across the ventricular chambers rendering volume overload. In the EuroScore II, risk assessment scale for cardiac surgery, VSR holds the maximum score on the scale (1).

Although excellent early reperfusion strategies for the myocardium following myocardial infarction (MI), have decreased the occurrence of VSR, the overall surgical mortality due to VSR reported in this paper is $38.9 \%$. Ventricular septal rupture is seen in the first week following MI. Myocardium differs from the rest of the tissues owing to the potential "limited blood supply to the damaged myocardium for healing due to narrowing of coronary arteries and the myocardium, which must continue to function during the process of repair, whereas other tissues can be immobilized". Most of the patients are elderly with hypertension leading to increased afterload to the left ventricle (LV), furthermore leading to stress at the junction of the healthy and infracted tissues, which allows the paradoxical systolic movement leading to the rupture of the necrosed myocardium $(2)$.

In the study, the data collected by the authors, indicate that early surgery in cases with VSR leads to mortality(1 to 30 days Vs $>30$ days: Odds ratio of $0.35 \mathrm{Vs} 0.25$ ) owing to technical difficulties, more fragile tissues, which leads to the suture to cut through the necrosed myocardium, should be operated as late as possible if hemodynamics permit.

The authors also highlight the importance of concomitant coronary artery bypass grafting $(\mathrm{CABG})(\mathrm{p}=0.544)$ and Mitral Valve Repair ( $\mathrm{p}=0.524)$ alongside VSR repair, adding that no additional risk was being imparted and it could prove to be beneficial to revascularize the weak myocardium in long run.

The study also mentions the role of percutaneous closure to bail out the crisis, pros \& cons, indications, success rate $(90 \%)$, and mortality.

In 1957, Denton Cooley first succeeded in surgical repair of VSR but unfortunately, the patient died 6 weeks later. The first successful long-term survivor was operated by Spencer Payne in 1960 by opening the right ventricular outflow tract (RVOT). The patient was asymptomatic $2 \frac{1}{2}$ yrs after surgery when followed up regularly (3). Early surgical repair of VSR following MI has drawbacks of its own, for example, suture cut through necrosed tissue. Via different approaches also, either through RV or LV, via direct suture closure, prosthetic closure, or percutaneous closure, outcomes are not satisfactory. Despite understanding the pathophysiology, early diagnosis, and revascularization, advances of cardiopulmonary bypass techniques and mechanical circulatory support like Intra Aortic Balloon Pump (IABP), ventricular assist devices, outcomes are dismal owing to the loss of functional myocardial reserve. A tailor can design the garment in the desired fashion, but can't change the quality of cloth, the same analogy is applied to the surgeon as well, till the quality of the myocardium is good.

\section{Conclusion:}

In summary, reading this paper gives provides an insight into the gravity of the scenario, possible interventions, and evident outcomes. The bottom line and what we most need is "functional myocardial reserve"

\section{References:}

1. Nashef SA, Roques F, Sharples LD, Nilsson J, Smith C, Goldstone AR, Lockowandt U. EuroSCORE II. Eur J Cardiothorac Surg. 2012 Apr;41(4):734-44; discussion 744-5. doi: 10.1093/ejcts/ezs043. Epub 2012 Feb 29. PMID: 22378855. 
2. Mallory GK, White PD, J. Salcedo-Salgar, The speed of healing of myocardial infarction: a study of the pathologic anatomy in seventy-two cases, Am. Heart J. 18 (6) (1939) 647-67

3. Payne WS, Hunt JC, Kirklin JW. Surgical Repair of Ventricular Septal Defect Due to Myocardial Infarction: Report of a Case. JAMA. 1963;183(7):603-605. doi:10.1001/jama.1963.63700070040020d 